\title{
AN EXAMPLE OF NECKPINCHING FOR RICCI FLOW ON
}

$$
S^{n+1}
$$

\author{
Sigurd Angenent And Dan Knopf
}

\begin{abstract}
We give an example of a class of metrics on $S^{n+1}$ that evolve under the Ricci Flow into a "neckpinch." We show that the solution has a Type I singularity, and that the length of the neck, i.e. the region where $|\mathrm{Rm}| \sim(T-t)^{-1}$, is bounded from below by $c \sqrt{(T-t)|\log (T-t)|}$ for some $c>0$.
\end{abstract}

\section{Preamble}

This paper is the first of two in which we study singularity formation in the Ricci flow. As motivation, consider a solution of the flow

$$
\begin{aligned}
\partial_{t} g & =-2 \operatorname{Rc}(g) \\
g(0) & =g_{0}
\end{aligned}
$$

starting from an arbitrary Riemannian manifold $\left(M^{m}, g_{0}\right)$. One should not be surprised if (1) becomes singular in finite time. Indeed, the simple estimate

$$
\partial_{t} R=\Delta R+2|\mathrm{Rc}|^{2} \geq \Delta R+\frac{2}{m} R^{2}
$$

for the evolution of the scalar curvature implies by the parabolic maximum principle that a finite-time singularity is inevitable if the curvature ever becomes everywhere positive.

In this context, it is very surprising indeed that no explicit examples of finitetime singularities were known until recently, except for trivial cases where the manifold is a product of constant-curvature factors, one of which vanishes all at once. The first rigorous examples [13] of finite-time singularities which occur on proper (compact) subsets of a manifold were constructed by Miles Simon. Here the manifold is a noncompact warped product $\mathbb{R} \times_{f} S^{n}$. These examples were obtained using upper barriers. Another family of examples was constructed in [4]. Here the manifold is the holomorphic line bundle $L^{-k}$ over $\mathbb{C P}^{n-1}$ with twisting number $k \in\{1, \ldots, n-1\}$, and the metric $g(t)$ is a complete $\mathrm{U}(n)$ invariant shrinking gradient Kähler-Ricci soliton. As $t \nearrow T<\infty$, the $\mathbb{C P}^{n-1}$ which constitutes the zero-section of the bundle disappears, while the metric $g(t)$ converges to a Kähler cone on the set $\left(\mathbb{C}^{n} \backslash\{0\}\right) / \mathbb{Z}_{k}$ which constitutes the

Recevied July 11, 2003.

First author partially supported by NSF grant DMS-0101124. Second author partially supported by NSF grant DMS-0328233. 
remainder of the bundle. No rigorous constructions of nontrivial finite-time singularitites on compact manifolds have yet appeared in the literature.

In sharp contrast to the scarcity of explicit examples, there is a substantial body of well-informed conjecture about the nature of Ricci flow singularities on compact manifolds. (See for instance Section 3 of [10].) In particular, it is strongly conjectured that curvature neckpinches should develop in finite time, where we use the following provisional definition of a neck pinch: A solution $\left(M^{n+1}, g(t)\right)$ to the Ricci flow which becomes singular at time $T<\infty$ has a neck pinch if there exist diffeomorphisms $\phi_{t}: \mathbb{R} \times S^{n} \rightarrow N(t)$ where $N(t)$ is some proper open subset of $M^{n+1}$ such that the metric $g(t)$ remains regular on $M^{n+1} \backslash N(t)$, and such that the pullback metric $\phi_{t}^{*}(g(t))$ approaches a "shrinking cylinder" metric

$$
d s^{2}+r(t)^{2} \cdot g_{\text {can }}
$$

in $C_{\text {loc }}^{\infty}$ on the cylinder $\mathbb{R} \times S^{n}$ as $t \nearrow T$, where $g_{\text {can }}$ is the round metric of radius 1 on $S^{n}$ and $\lim _{t \nearrow T} r(t)=0$.

Except for a sphere shrinking to a round point, the neckpinch is topologically the simplest singularity which the Ricci flow can encounter. Arguably, it is also the most important, at least with respect to the goal of obtaining topological information from the Ricci flow. Indeed, much effort has been exerted to understand singularity formation (especially in dimensions three and four) by the strategy of forming sequences of parabolic dilations at a developing singularity. Such sequences $\left(M^{n+1}, g_{j}(t)\right)$ are defined by

$$
g_{j}(t):=\lambda_{j} g\left(t_{j}+\frac{t}{\lambda_{j}}\right), \quad-\lambda_{j} t_{j} \leq t<\lambda_{j}\left(T-t_{j}\right),
$$

where $t_{j} \nearrow T$ and $\lambda_{j} \nearrow \infty$. In order to obtain information from the Ricci flow about the geometry of the original manifold near the singularity and just prior to its formation, one studies the properties of limits of these dilations. (See for example $[10,11]$ and the recent articles $[14,15]$.)

The present paper and its successor are not directly relevant to topological applications of the Ricci flow, however. Rather, they are inspired by the long tradition of singularity analysis for nonlinear PDE and geometric evolution equations.

In this paper, we demonstrate the existence of neckpinch singularities on compact Riemannian manifolds, in particular for an open set of rotationallysymmetric initial metrics on topological spheres $S^{n+1}$. Our main result is the following:

Theorem 1.1. If $n \geq 2$, there exists an open subset of the family of metrics on $S^{n+1}$ possessing $\mathrm{SO}(n+1)$ symmetries such that the Ricci flow starting at any metric in this set develops a neckpinch at some time $T<\infty$. The singularity is rapidly-forming (Type I), and any sequence of parabolic dilations formed at the developing singularity converges to a shrinking cylinder soliton

$$
d s \otimes d s+2(n-1)(T-t) g_{\text {can }} .
$$


This convergence takes place uniformly in any ball of radius

$$
o\left(\sqrt{(T-t) \log \frac{1}{T-t}}\right)
$$

centered at the neck.

Furthermore, there exist constants $0<\delta, C<\infty$ such that the radius $\psi$ of the sphere at distance $\sigma$ from the neck pinch is bounded from above by

$$
\psi \leq \sqrt{2(n-1)(T-t)}+\frac{C \sigma^{2}}{-\log (T-t) \sqrt{T-t}}
$$

for $|\sigma| \leq 2 \sqrt{-(T-t) \log (T-t)}$, and

$$
\psi \leq C \frac{\sigma}{\sqrt{-\log (T-t)}} \sqrt{\log \frac{\sigma}{\sqrt{-(T-t) \log (T-t)}}}
$$

for $2 \sqrt{-(T-t) \log (T-t)} \leq \sigma \leq(T-t)^{\frac{1}{2}-\delta}$.

The class of initial metrics for which we establish "neck pinching" is essentially described by three conditions: (i) the initial metric should have positive scalar curvature, (ii) the sectional curvature of the initial metric should be positive on planes tangential to the spheres $\{x\} \times S^{n}$, and (iii) the initial metric should be "sufficiently pinched." See Section 8 for details. One difference between our approach and Simon's [13], is that Simon assumes that the Ricci curvature of the meridians $\mathbb{R} \times\{p\}\left(p \in S^{n}\right)$ is negative, which can happen if the manifold $M^{n+1}$ is a cylinder $\mathbb{R} \times S^{n}$, but not if $M^{n+1}$ is a sphere $S^{n+1}$.

Finally, in Section 10, we consider the special case of a reflection-symmetric metric with one neck. In this situation we prove that the singularity occurs only on the totally geodesic $S^{n}$ which constitutes the equator, provided the diameter of $\left(M^{n+1}, g(t)\right)$ remains bounded as $t \nearrow T$. It is not clear to us that this diameter must indeed always remain bounded. However, a more detailed analysis of the asymptotics of the neckpinch, which we present in a subsequent paper, does show that this hypothesis is met for a subset of the solutions considered here.

The results obtained in the present paper strongly resemble familiar theorems for the rotationally symmetric mean curvature flow. There is a vast body of work on singularity formation for that flow. A pioneering paper in the rotationally symmetric case is [12], and a more general approach to such singularities is considered in [2]. Aspects of the argument for the Ricci flow are significantly more difficult than the corresponding arguments for the mean curvature flow, however, in part because one must work much harder in the present case to control the solution on the "polar caps." (See Section 5.4.)

In the successor to this paper, we will remove the hypothesis of rotational symmetry and thereby derive formal matched asymptotics for fully general neckpinch singularities. We will also develop more detailed asymptotics for the rotationallysymmetric case. 
These extend and generalize the results obtained in Section 9 below, and indicate that the error bounds in Lemma 9.4 are sharp. Examples of this sort of analysis for the semilinear reaction-diffusion equation $u_{t}=\Delta u+u^{p}$ can be found in $[6,7,8]$ and [5]. Rigorous matched asymptotics for slowly-forming singularities of the mean curvature flow were developed in [3].

Acknowledgement. The authors wish to express their gratitude for the hospitality and partial support provided by the National Center for Theoretical Sciences in Hsinchu, Taiwan. It was during their 2002 summer Workshop on Geometric Evolution Equations that the original observations in this paper were made. The authors also wish to thank Miles Simon for several stimulating conversations in Hsinchu.

\section{The equations}

We consider metrics on $S^{n+1}$ given by

$$
g=\varphi(x)^{2} d x \otimes d x+\psi(x)^{2} \hat{g},
$$

in which $\hat{g} \equiv g_{\text {can }}$ is the metric of constant curvature 1 on $S^{n}$. We have punctured the sphere $S^{n+1}$ at its north and south poles $P_{ \pm}$, and identified the remaining manifold with $(-1,1) \times S^{n}(1)$, with $x$ the coordinate on $(-1,1)$ and $S^{n}(1)$ the unit sphere.

2.1. Coordinates. The coordinate $x$ is ungeometric: a more geometric quantity is the distance $s$ to the equator given by

$$
s(x)=\int_{0}^{x} \varphi(x) d x .
$$

One could introduce $s$ as a new coordinate, but for logical consistency we will adopt the following convention: in this paper all functions (tensors, forms, etc.) defined on $S^{n+1} \backslash$ north \& south poles\} will be functions of the $x$ variable. Whenever we write a relation of the type $f=f(s)$, it is to be understood as shorthand for $f=f(s(x))$ (or $f=f(s(x, t)$ ) for evolving metrics).

The "derivative with respect to $s$ " is given by

$$
\frac{\partial}{\partial s}=\frac{1}{\varphi(x)} \frac{\partial}{\partial x} \text {. }
$$

We also define

$$
d s=\varphi(x) d x
$$

even when the metric evolves, in which case standard notation would have suggested $d s(x, t)=s_{x} d x+s_{t} d t$ instead.

With this notation the metric is

$$
g=(d s)^{2}+\psi^{2} \hat{g}
$$


2.2. Curvature tensors. The Riemann tensor is completely determined by the sectional curvatures of the 2-planes perpendicular to the spheres $\{x\} \times S^{n}$, and the 2-planes tangential to these spheres. These curvatures are (respectively)

$$
K_{0}=-\frac{\psi_{s s}}{\psi}, \quad K_{1}=\frac{1-\psi_{s}^{2}}{\psi^{2}} .
$$

In the ungeometric coordinate $x$ the Ricci tensor of the metric $g$ given by (4) is

$\mathrm{Rc}=n\left\{-\frac{\psi_{x x}}{\psi}+\frac{\varphi_{x} \psi_{x}}{\psi \varphi}\right\}(d x)^{2}+\left\{-\frac{\psi \psi_{x x}}{\varphi^{2}}-\frac{(n-1) \psi_{x}^{2}}{\varphi^{2}}+\frac{\psi \varphi_{x} \psi_{x}}{\varphi^{3}}+n-1\right\} \hat{g}$.

In the geometric coordinate this simplifies to

$$
\begin{aligned}
\mathrm{Rc} & =n K_{0}(d s)^{2}+\left[K_{0}+(n-1) K_{1}\right] \psi^{2} \hat{g} \\
& =-n \frac{\psi_{s s}}{\psi}(d s)^{2}+\left\{-\psi \psi_{s s}-(n-1) \psi_{s}^{2}+n-1\right\} \hat{g} .
\end{aligned}
$$

The scalar curvature is given by

$$
\begin{aligned}
R & =g^{j k} R_{j k} \\
& =n K_{0}+n\left[K_{0}+(n-1) K_{1}\right] \\
& =2 n K_{0}+n(n-1) K_{1} \\
& =n\left\{-2 \frac{\psi_{s s}}{\psi}+(n-1) \frac{1-\psi_{s}^{2}}{\psi^{2}}\right\} .
\end{aligned}
$$

2.3. Evolution equations. Suppose we have a time dependent family of metrics $g(\cdot, t)$ which evolves by the Ricci flow (1). Then the "radius" $\psi(x, t)$ will satisfy

$$
\partial_{t} \psi=\psi_{s s}-(n-1) \frac{1-\psi_{s}^{2}}{\psi} .
$$

The quantity $\varphi(x, t)$ evolves by

$$
\partial_{t} \varphi=n\left\{\frac{\psi_{x x}}{\varphi \psi}-\frac{\varphi_{x} \psi_{x}}{\psi \varphi^{2}}\right\}=n \frac{\psi_{s s}}{\psi} \varphi
$$

The equations (7)-(11) can also be found in [13] (Propositions 2.1 and 4.1).

\section{Derived equations}

3.1. Equations for powers of $\psi$. Various powers of $\psi$ satisfy equations similar to (10). If we set $u=\psi^{k}$ for any $k \neq 0$, then

$$
\begin{aligned}
u_{t} & =k \psi^{k-1} \psi_{t} \\
& =k \psi^{k-1} \psi_{s s}+(n-1) k \psi^{k-2} \psi_{s}^{2}-(n-1) k \psi^{k-2} \\
& =u_{s s}+\frac{n-k}{k} \frac{u_{s}^{2}}{u}-(n-1) k u^{1-\frac{2}{k}}
\end{aligned}
$$


In particular, for $k=n$ we get

$$
u_{t}=u_{s s}-n(n-1) u^{1-\frac{2}{n}} .
$$

For $k=2$ we get

$$
u_{t}=u_{s s}+\frac{n-2}{2} \frac{u_{s}^{2}}{u}-2(n-1) .
$$

3.2. Equations for derivatives of $\psi$. The partial derivatives $\partial_{t}$ and $\partial_{x}$ commute, but $\partial_{t}$ and $\partial_{s}$ do not. To commute them, we must use the following identity

$$
\left[\partial_{t}, \partial_{s}\right]=\left[\partial_{t}, \frac{1}{\varphi(x, t)} \partial_{x}\right]=\frac{-\varphi_{t}}{\varphi^{2}} \partial_{x}=-n \frac{\psi_{s s}}{\psi} \partial_{s}
$$

Thus we find

$$
\begin{aligned}
\partial_{t}\left(\psi_{s}\right) & =\partial_{s}\left(\psi_{t}\right)+\left[\partial_{t}, \partial_{s}\right] \psi \\
& =\partial_{s s s} \psi+(n-2) \frac{\psi_{s} \psi_{s s}}{\psi}+(n-1) \frac{1-\psi_{s}^{2}}{\psi^{2}} \psi_{s}
\end{aligned}
$$

that is, the quantity $v=\psi_{s}$ satisfies the heat equation

$$
\partial_{t} v=v_{s s}+\frac{n-2}{\psi} v v_{s}+\frac{n-1}{\psi^{2}}\left(1-v^{2}\right) v .
$$

For $w=\psi_{\text {ss }}$ we find after a similar computation

$$
\begin{aligned}
\partial_{t} w=w_{s s} & +(n-2) \frac{\psi_{s}}{\psi} w_{s}-2 \frac{w^{2}}{\psi}-(4 n-5) \frac{\psi_{s}^{2}}{\psi^{2}} w+\frac{n-1}{\psi^{2}} w \\
& -2(n-1) \frac{\psi_{s}^{2}\left(1-\psi_{s}^{2}\right)}{\psi^{3}}
\end{aligned}
$$

i.e.

$$
\begin{aligned}
\partial_{t}\left(\psi_{s s}\right)=\left(\psi_{s s}\right)_{s s}+(n-2) \frac{\psi_{s}}{\psi}\left(\psi_{s s}\right)_{s} & \\
- & 2 \frac{\psi_{s s}^{2}}{\psi}-(4 n-5) \frac{\psi_{s}^{2}}{\psi^{2}} \psi_{s s}+\frac{n-1}{\psi^{2}} \psi_{s s} \\
& -2(n-1) \frac{\psi_{s}^{2}\left(1-\psi_{s}^{2}\right)}{\psi^{3}} .
\end{aligned}
$$

We also have equations for $\psi_{t}$ and $K=\psi_{s s} / \psi\left(=-K_{0}\right)$, namely

$$
\begin{aligned}
\partial_{t}\left(\psi_{t}\right)=\left(\psi_{t}\right)_{s s} & +(n-2) \frac{\psi_{s}}{\psi}\left(\psi_{t}\right)_{s} \\
- & \frac{2 n}{\psi} \psi_{t}^{2}+(2 n-1)^{2} \frac{\psi_{s}^{2}}{\psi^{2}} \psi_{t}-\frac{(4 n-1)(n-1)}{\psi^{2}} \psi_{t} \\
& -2 n(n-1) \frac{1-\psi_{s}^{2}}{\psi^{3}}\left[(n-1)\left(1-\psi_{s}^{2}\right)+\psi_{s}^{2}\right]
\end{aligned}
$$


and

$$
\begin{aligned}
\partial_{t} K= & K_{s s}+n \frac{\psi_{s}}{\psi} K_{s}-2 K^{2} \\
& -4(n-1) \frac{\psi_{s}^{2}}{\psi^{2}} K+\frac{2(n-1)}{\psi^{2}} K-2(n-1) \frac{\psi_{s}^{2}}{\psi^{4}}\left(1-\psi_{s}^{2}\right) .
\end{aligned}
$$

Another way of deriving these equations is based on the observation that the Ricci tensor is given by

$$
\mathrm{Rc}=-n \frac{\psi_{s s}}{\psi}(d s)^{2}-\psi \psi_{t} \hat{g}
$$

so that the two quantities $-n K$ and $-\psi \psi_{t}$ are the eigenvalues of the Ricci tensor. Under Ricci flow, this tensor evolves by

$$
\partial_{t}(\mathrm{Rc})_{j k}=(\Delta \mathrm{Rc})_{j k}+2(\mathrm{Rm})_{p j k q}(\mathrm{Rc})^{p q}-2(\mathrm{Rc})_{j}^{p}(\mathrm{Rc})_{p k},
$$

where all contractions are done with respect to the evolving metric $g$. (See Section 5.4 below.)

\subsection{Curvature Pinching. Consider}

$$
a=\psi \psi_{s s}-\psi_{s}^{2}+1=\psi^{2}\left(K_{1}-K_{0}\right) .
$$

This quantity provides a scale invariant measure for the difference of the two sectional curvatures $K_{0}$ and $K_{1}$.

Lemma 3.1. The quantity a evolves by

$$
a_{t}=a_{s s}+(n-4) \frac{\psi_{s}}{\psi} a_{s}-4(n-1) \frac{\psi_{s}^{2}}{\psi^{2}} a
$$

Proof. Note that

$$
a=\psi w-v^{2}+1
$$

where $v=\psi_{s}$ and $w=\psi_{s s}$ are defined above in Section 3.2. One computes that

$$
a_{s}=\psi_{s} w+\psi w_{s}-2 v v_{s}=\psi w_{s}-v w
$$

and

$$
a_{s s}=\psi_{s} w_{s}+\psi w_{s s}-v_{s} w-v w_{s}=\psi w_{s s}-w^{2}
$$


Then recalling equation (10) for the evolution of $\psi$, equation (16) for the evolution of $v$, and equation (17) for the evolution of $w$, one derives the equation

$$
\begin{aligned}
a_{t}= & w \psi_{t}+\psi w_{t}-2 v v_{t} \\
= & w\left[w-(n-1) \frac{1-v^{2}}{\psi}\right] \\
& +\psi\left[\begin{array}{c}
w_{s s}+(n-2) \frac{v w_{s}}{\psi}-2 \frac{w^{2}}{\psi}-(4 n-5) \frac{v^{2} w}{\psi^{2}} \\
+(n-1) \frac{w}{\psi^{2}}-2(n-1) \frac{v^{2}\left(1-v^{2}\right)}{\psi^{3}}
\end{array}\right] \\
& -2 v\left[w_{s}+(n-2) \frac{v w}{\psi}+(n-1) \frac{v\left(1-v^{2}\right)}{\psi^{2}}\right] \\
= & \psi w_{s s}-w^{2}+(n-4) v w_{s}-(5 n-8) \frac{v^{2} w}{\psi}-4(n-1) \frac{v^{2}\left(1-v^{2}\right)}{\psi^{2}} \\
= & a_{s s}+(n-4) \frac{v}{\psi} a_{s}-4(n-1) \frac{v^{2}}{\psi^{2}} a .
\end{aligned}
$$

Applying the maximum principle immediately yields the following estimate.

Corollary 3.2. $\sup |a(\cdot, t)| \leq \sup |a(\cdot, 0)|$.

3.4. Curvature Splitting. The following evolution equations will be useful when we consider the asymptotics of a neck pinch. We recall that we earlier defined $K=-K_{0}=\psi_{s s} / \psi$, and we now write $L=K_{1}$. With this notation, one can write the evolution equation (20) for $K$ as

$$
K_{t}=\Delta K+2(n-1) K L-2 K^{2}-2(n-1) \frac{\psi_{s}^{2}}{\psi^{2}}(K+L),
$$

since the Laplacian of a radially symmetric function is given by

$$
\Delta f=\frac{\partial^{2} f}{\partial s^{2}}+n \frac{\psi_{s}}{\psi} \frac{\partial f}{\partial s} .
$$

For $L$ we have:

Proposition 3.3. If $g(\cdot, t)=\varphi^{2}(d x)^{2}+\psi^{2} \hat{g}$ is a solution to the Ricci flow, then $L$ evolves by

$$
\begin{aligned}
L_{t} & =\Delta L+2 \frac{\psi_{s}}{\psi} L_{s}+2\left[K^{2}+(n-1) L^{2}\right] \\
& =\Delta L-4 \frac{\psi_{s}^{2}}{\psi^{2}}(K+L)+2\left[K^{2}+(n-1) L^{2}\right] .
\end{aligned}
$$

Proof. Using equations (10) and (16), one computes that

$$
\begin{aligned}
L_{t} & =-2 \frac{\psi_{s}}{\psi^{2}}\left(\psi_{s}\right)_{t}-2 \frac{L}{\psi} \psi_{t} \\
& =-2 \frac{\psi \psi_{s s s}}{\psi^{2}}-2(n-1) \frac{\psi_{s}^{2}}{\psi^{2}}(K+L)+2\left(\frac{\psi_{s}^{2}}{\psi^{2}}-L\right) K+2(n-1) L^{2} .
\end{aligned}
$$


Then observing that

$$
L_{s}=-2 \frac{\psi_{s}}{\psi}(K+L) \text {, }
$$

one calculates

$$
L_{s s}=-2 \frac{\psi \psi_{s s s}}{\psi^{2}}+6 \frac{\psi_{s}^{2}}{\psi^{2}}(K+L)+2\left(\frac{\psi_{s}^{2}}{\psi^{2}}-L\right) K-2 K^{2} .
$$

Combining these equations yields

$$
L_{t}=L_{s s}-2(n+2) \frac{\psi_{s}^{2}}{\psi^{2}}(K+L)+2\left[K^{2}+(n-1) L^{2}\right],
$$

whence the result follows.

Corollary 3.4. $L_{\min }(t)$ is nondecreasing; and whenever $L_{\min }(0) \neq 0$, one has

$$
L_{\min }(t) \geq \frac{1}{L_{\min }(0)^{-1}-2(n-1) t} .
$$

Now consider

$$
F=\frac{K}{L} \log L
$$

Then using equations (22) and (23a), one computes the following for $F$.

Proposition 3.5. If $g(\cdot, t)=\varphi^{2}(d x)^{2}+\psi^{2} \hat{g}$ is a solution to the Ricci flow with $L>1$, then $F$ evolves by

$$
\begin{aligned}
F_{t}=\Delta F+2\left(\frac{\log L-1}{L \log L}\right) L_{s} F_{s}+\left(\frac{2-\log L}{\log L}\right) \frac{K L_{s}^{2}}{L^{3}} & \\
& -2 P\left(\frac{\psi_{s}}{\psi}\right)^{2} \frac{K+L}{L}+2 Q K
\end{aligned}
$$

where

$$
P=(n-1) \log L-2 \frac{K}{L}(\log L-1)
$$

and

$$
Q=n-1-\frac{K^{2}}{L^{2}}(\log L-1)-F
$$

The hypothesis $L>1$ is needed here to keep $\log L$ positive, and in particular nonzero. 


\section{Boundary conditions}

All quantities considered here are defined for $-1<x<1$. The "radius" $\psi(x, t)$ must vanish at $x= \pm 1$, for otherwise the manifold would fail to be a sphere. At these endpoints the derivative of $\psi$ is also prescribed.

Proposition 4.1. Let $g=\varphi(x)^{2}(d x)^{2}+\psi(x)^{2} \hat{g}$ be a smooth Riemannian metric on $(-1,1) \times S^{n}$ which extends to a smooth metric on $S^{n+1}$. Then

$$
\lim _{x \rightarrow \pm 1} \psi_{s}=\mp 1 \text {. }
$$

Write $\bar{s}=\int_{0}^{1} \varphi(x) d x$ for the distance from the equator $x=0$ to the north pole $x=1$. Then $\psi /(\bar{s}-s)$ extends to a smooth even function of $\bar{s}-s$, so that

$$
\psi=(\bar{s}-s)+A_{3}(\bar{s}-s)^{3}+\cdots+A_{2 m+1}(\bar{s}-s)^{2 m+1}+\cdots .
$$

Proof. The quantity $\bar{s}-s$ is the distance from any point on $\{x\} \times S^{n}$ to the north pole. Thus the expression $g=(d s)^{2}+\psi^{2} \hat{g}$ is a representation of the metric $g$ in geodesic polar coordinates, with $\bar{s}-s$ being the radius. In this light, the proposition is a standard result from Riemannian geometry.

\section{The shape of the solution}

\subsection{Derivative estimates.}

Proposition 5.1. Assume $g(\cdot, t)=\varphi^{2}(d x)^{2}+\psi^{2} \hat{g}$ is a solution to the Ricci flow for $0 \leq t<T$. Then

$$
1 \leq \sup \left|\partial_{s} \psi(\cdot, t)\right| \leq \sup \left|\partial_{s} \psi(\cdot, 0)\right| .
$$

Proof. One applies the maximum principle to (16). This equation says that at any maximum of $\psi_{s}$ which exceeds 1 , one has

$$
\partial_{t} \psi_{s} \leq \frac{n-1}{\psi^{2}}\left(1-\psi_{s}^{2}\right) \psi_{s}<0
$$

Similarly, at any minimum of $\psi_{s}$ with $\psi_{s}<-1$, one has $\partial_{t} \psi_{s}>0$. The result follows once we observe that by Proposition 4.1, $g$ extends to a smooth metric on $S^{n+1}$ only if $\sup \left|\partial_{s} \psi(\cdot, 0)\right| \geq 1$.

Proposition 5.2. Assume $g(\cdot, t)=\varphi^{2}(d x)^{2}+\psi^{2} \hat{g}$ is a solution to the Ricci flow for $0 \leq t<T$ with $\left|\psi_{s}\right| \leq 1$ and initially positive scalar curvature. Then $R>0$ and $\partial_{t} \psi<0$ on $S^{n+1} \times[0, T)$.

Proof. The scalar curvature satisfies $R_{t}=\Delta R+2|\mathrm{Rc}|^{2} \geq \Delta R$, so positivity of $R$ is preserved.

Our assumption $\left|\psi_{s}\right| \leq 1$ forces the sectional curvature $K_{1}=\psi^{-2}\left(1-\psi_{s}^{2}\right)$ to be nonnegative everywhere. If at any point one has $\psi_{s s} \leq 0$, then $K_{0}=$ $-\psi_{s s} / \psi \geq 0$, and hence $\psi_{t}=\psi_{s s}-(n-1)\left(1-\psi_{s}^{2}\right) / \psi<0$. If on the other hand $\psi_{s s}>0$, then $K_{0}=-\psi_{s s} / \psi<0$, so that

$$
-\psi_{t}=\psi\left(K_{0}+(n-1) K_{1}\right)=\psi\left(\frac{R}{n}-K_{0}\right)>0
$$


as claimed.

Proposition 5.3. If $\left|\psi_{s}\right| \leq 1$, then $\left|\partial_{t}\left(\psi^{2}\right)\right| \leq 2 n+2 \sup |a(\cdot, 0)|$, where $a=$ $\psi \psi_{s s}-\psi_{s}^{2}+1$, as in $\S 3.3$.

Proof. We showed in Corollary 3.2 that $|a| \leq \sup |a(\cdot, 0)|$. By definition one has $\psi_{s s}=\left(a+\psi_{s}^{2}-1\right) / \psi$, so that

$$
\psi_{t}=\psi_{s s}-(n-1)\left(1-\psi_{s}^{2}\right) / \psi=\frac{a-n\left(1-\psi_{s}^{2}\right)}{\psi},
$$

from which the proposition immediately follows.

\subsection{The limit at $t=T$.}

Proposition 5.4. Assume $g=\varphi(x, t)^{2}(d x)^{2}+\psi(x, t)^{2} \hat{g}$ is a solution of the Ricci flow which at $t=0$ satisfies $\left|\psi_{s}\right| \leq 1$. Then $\psi(x, T):=\lim _{t \nearrow T} \psi(x, t)$ exists.

Proof. The condition $\left|\psi_{s}\right| \leq 1$ is preserved by Ricci flow. Hence by Proposition $5.3, \psi(x, t)^{2}$ is a uniformly Lipschitz continuous function of time. The limit $\lim _{t \nearrow T} \psi(x, t)$ must therefore exist for each $x \in[-1,1]$.

5.3. Necks and Bumps. The derivative $v=\psi_{s}$ satisfies (16), which we can write as a linear parabolic equation

$$
\partial_{t} v=v_{s s}+Q v
$$

with

$$
Q=(n-2) \frac{\psi_{s s}}{\psi}+(n-1) \frac{1-\psi_{s}^{2}}{\psi} .
$$

Using $\partial_{s}=\varphi^{-1} \partial_{x}$, one can write this equation as

$$
\partial_{t} v=\varphi^{-1}\left(\varphi^{-1} v_{x}\right)_{x}+Q(x, t) v=A(x, t) v_{x x}+B(x, t) v_{x}+C(x, t) v
$$

for suitable coefficients $A, B$, and $C$. Furthermore, at the extremes $x= \pm 1$ we have seen that $\psi_{s} \rightarrow \mp 1$. The Sturmian theorem [1] therefore applies, and we conclude the following.

Lemma 5.5. At any time $t \in(0, T)$, the derivative $v=\psi_{s}(\cdot, t)$ has a finite number of zeroes, as a function of $x \in(-1,1)$. This number of zeroes is nonincreasing in time, and at any moment $t_{0} \in(0, T)$ that $\psi(\cdot, t)$ has a multiple zero (i.e. a point at which $\psi_{s}=\psi_{\text {ss }}=0$ simultaneously) the number of zeroes of $\psi_{s}(\cdot, t)$ drops.

We will refer to local maxima of $x \mapsto \psi(x, t)$ as bumps, and local minima as necks. The lemma says that during any evolution by the Ricci flow of $g(t)=$ $\varphi^{2}(d x)^{2}+\psi^{2} \hat{g}$, the number of necks cannot increase with time, while all necks and bumps must be nondegenerate maxima/minima, except when one or more bumps and necks come together and annihilate each other. In particular, if the number of necks does not change, then all necks and bumps are nondegenerate. 


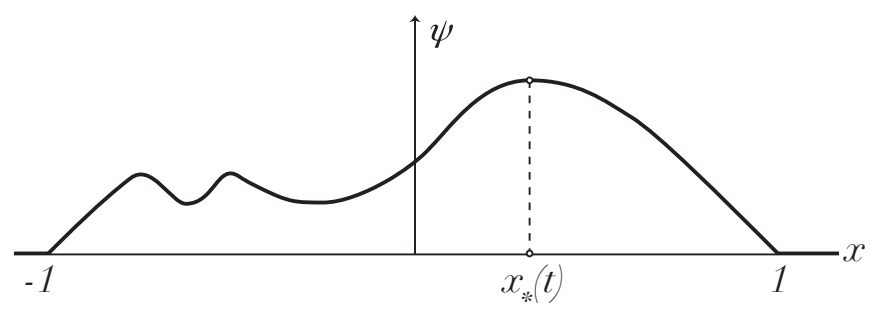

Figure 1. A Genetically Modified Peanut

5.4. Preserving the polar caps. In this section, we prove that the curvature remains positive on the polar caps if it is so initially. Let $x_{*}(t) \in(0,1)$ denote the location of the right-most bump, namely the largest $x \in(-1,1)$ at which $\psi_{x}(x, t)=0$. Then the "polar cap" of $S^{n+1}$ is the set $\left(\left(x_{*}(t), 1\right) \times S^{n}(1)\right) \cup\left\{P_{+}\right\}$.

Lemma 5.6. If $\psi_{s s}(x, 0) \leq 0$ for $x_{*}(0)<x<1$, then $\psi_{s s}(x, t) \leq 0$ for $x_{*}(t)<$ $x<1$ and all $0<t<T$.

To prove the lemma, we shall use the following slight modification of the tensor maximum principle introduced in [9].

Proposition 5.7. Let $\left\{\mathcal{N}_{t}, \partial \mathcal{N}_{t}, g(t): 0 \leq t<T\right\}$ be a smooth 1-parameter family of compact Riemannian manifolds with boundary. Let $S$ and $F$ be symmetric $(2,0)$-tensor fields on $\mathcal{N}_{t}$ such that $S$ evolves by

$$
\frac{\partial}{\partial t} S \geq \Delta S+F * S,
$$

where $F * S$ denotes the symmetrized product $(F * S)_{i j}=F_{i}^{k} S_{k j}+S_{i}^{k} F_{k j}$. Suppose that $\inf _{p \in \mathcal{N}_{t}} S(p, 0) \geq 0$ and that $S(q, t) \geq 0$ for all points $q \in \partial \mathcal{N}_{t}$ and times $t \in[0, T)$. If $(F * S)(V, \cdot) \geq 0$ whenever $S(V, \cdot)=0$, then

$$
\inf _{p \in \mathcal{N}_{t}, t \in[0, T)} S(p, t) \geq 0 .
$$

If all inequalities are strict then the proof is entirely standard, once we note that the hypotheses imply that $S$ can first attain a zero eigenvalue only at an interior point of $\mathcal{N}_{t}$. To reduce the general case to the case of strict inequalities one considers $\tilde{S}_{i j}=S_{i j}+\varepsilon e^{\lambda t} g_{i j}(t)$ for large enough $\lambda>0$ and arbitrary small $\varepsilon>0$.

Proof of Lemma 5.6. First we show that the Ricci tensor satisfies

$$
\frac{\partial}{\partial t} \mathrm{Rc}=\Delta \mathrm{Rc}+F * \mathrm{Rc}
$$

where $F$ is the $(2,0)$ tensor given by

$$
F=\left(K_{1}-K_{0}\right)\left[(n-1)(d s)^{2}+\psi^{2} \hat{g}\right] .
$$


To verify $(27)$ at any given point $(x, P) \in(-1,1) \times S^{n}$ we choose coordinates $\left\{y^{1}, \ldots, y^{n}\right\}$ near $P$ on $S^{n}$ in which the standard metric $\hat{g}$ has components $\hat{g}_{\alpha \beta}=\delta_{\alpha \beta}$ at $P$.

We set $y^{0}=s$ so that $\left\{y^{0}, y^{1}, \ldots, y^{n}\right\}$ is a coordinate system near $(x, P)$ on $(-1,1) \times S^{n}$, and adopt the convention that Roman indices belong to $\{0, \ldots n\}$ while Greek indices belong to $\{1, \ldots, n\}$. The only nonvanishing components of the metric $g$ in these coordinates are $g_{00}=1$ and $g_{\alpha \alpha}=\psi^{2} \hat{g}_{\alpha \alpha}$. Observe that all components of the Riemann tensor $\mathrm{Rm}=\left(R_{i j k \ell}\right)$ vanish in these coordinates except $R_{\alpha 00 \alpha}=\psi^{2} K_{0}$ and $R_{\alpha \beta \beta \alpha}=\psi^{4} K_{1} \quad(\alpha \neq \beta)$. Similarly, all components of the Ricci tensor $\mathrm{Rc}=\left(R_{i j}\right)$ vanish except $R_{00}=n K_{0}$ and $R_{\alpha \alpha}=\psi^{2}\left[K_{0}+(n-1) K_{1}\right]$. The evolution equation (27) now follows from (21).

We have established $(27)$ on the punctured sphere $(-1,1) \times S^{n}$. By continuity it remains valid at the poles $\{ \pm 1\} \times S^{n}$.

To apply Proposition 5.7, let $\mathcal{N}_{t}$ denote the topological $(n+1)$-ball

$$
\mathcal{N}_{t}=\left\{(x, p): x \geq x_{*}(t), p \in S^{n}\right\}
$$

endowed with the metric $g(t)$. Observe that $K_{0}$ and $K_{1}$ are strictly positive on

$$
\partial \mathcal{N}_{t}=\left\{x_{*}(t)\right\} \times S^{n},
$$

because $\psi$ has a local maximum at $x_{*}(t)$, and $\psi_{s}$ has a simple zero there by Lemma 5.5. So Rc $>0$ on $\partial \mathcal{N}_{t}$. If $\psi(\cdot, 0)$ is strictly convex for all $x \geq x_{*}(0)$, then $\operatorname{Rc}(\cdot, 0)>0$ on $\mathcal{N}_{t}$. So if Rc ever acquires a zero eigenvalue, it must do so at some point $p \in \operatorname{int} \mathcal{N}_{t}$ and time $t \in(0, T)$. If $\left.\operatorname{Rc}(V, V)\right|_{(p, t)}=0$ for some vector $V \in T_{p} \mathcal{N}_{t}$, then $(F * \mathrm{Rc})(V, V)=0$, because $F$ and Rc commute. Hence Proposition 5.7 implies that $\mathrm{Rc} \geq 0$ on $\mathcal{N}_{t}$ for as long as $g(t)$ exists. Lemma 5.6 follows immediately.

\section{Shrinking rate of a neck}

In this section we consider a solution $g(t)=(d s)^{2}+\psi(x, t)^{2} \hat{g}$ of Ricci flow, and we define

$$
r_{\min }(t)=\min \left\{\psi(x, t) \mid \psi_{x}(x, t)=0\right\}
$$

i.e., $r_{\min }(t)$ is the radius of the smallest neck of the solution at time $t$. If the solution has no necks, then $r_{\min }(t)$ will be undefined.

Lemma 6.1. Let $g(t)=(d s)^{2}+\psi(x, t)^{2} \hat{g}$ be a solution of Ricci flow with $R \geq 0$. Then

$$
(n-1)(T-t) \leq r_{\min }(t)^{2} \leq 2(n-1)(T-t)
$$

An important direct consequence of this Lemma is that any initial metric generates a solution which must either become singular at or before

$$
T=\frac{r_{\min }(0)^{2}}{n-1}
$$

or else lose all its necks before this time so that $r_{\min }(t)$ becomes undefined. 
Proof. For all but a finite number of times $\psi(\cdot, t)$ will be a Morse function, and its critical values will be smooth functions of time. The smallest critical value, i.e. $r_{\min }(t)$, is therefore a Lipschitz continuous function of time. We will show that

$$
-\frac{n-1}{r_{\min }(t)} \leq r_{\min }^{\prime}(t) \leq-\frac{n-1}{2 r_{\min }(t)}
$$

holds for almost all $t$. After integration this implies the Lemma.

Suppose that at some time $t_{0}$ the function $\psi\left(\cdot, t_{0}\right)$ is a Morse function, and let its smallest critical value be attained at $x\left(t_{0}\right)$. Then by the Implicit Function Theorem there is a smooth function $x(\cdot)$ defined near $t=t_{0}$, such that $\psi_{x}(x(t), t)=0$. One has

$$
\begin{aligned}
\frac{d \psi(x(t), t)}{d t} & =\psi_{t}(x(t), t)+\psi_{x}(x(t), t) x^{\prime}(t) \\
& =\psi_{t}(x(t), t) \\
& =\psi_{s s}(x(t), t)-\frac{n-1}{r_{\min }(t)} .
\end{aligned}
$$

The first inequality in (29) follows immediately from (30) once one realizes that at a neck one has $\psi_{s s} \geq 0$.

To get the other inequality we recall that the scalar curvature satisfies $R=$ $2 n K_{0}+n(n-1) K_{1}$, where $K_{0}=-\psi_{s s} / \psi$ and $K_{1}=\left(1-\psi_{s}^{2}\right) / \psi^{2}$. At a neck, we therefore have

$$
\psi_{s s}=-\psi K_{0}=\frac{n-1}{2 \psi}-\frac{R}{2 n} \psi
$$

Since $R>0$ holds on our solution, we find

$$
\frac{d \psi(x(t), t)}{d t} \leq-\frac{n-1}{2 \psi}
$$

whence the second inequality in (29).

We note that the hypothesis $R>0$ is not really necessary in this Lemma. If one assumes $R \geq-C$ for some constant (which is always the case, since $R_{\min }$ is nondecreasing in time) then one finds

$$
-\frac{n-1}{r_{\min }(t)} \leq r_{\min }^{\prime}(t) \leq-\frac{n-1}{2 r_{\min }(t)}+\frac{C}{2 n} r_{\min }(t)
$$

From this one deduces $\epsilon(T-t) \leq r_{\min }(t)^{2} \leq 2(n-1)(T-t)$.

\section{The caps stay smooth}

In this section, we prove that no singularity occurs on the polar caps (i.e., they don't melt).

Lemma 7.1. There is a constant $C$, depending on the solution $g(t)$ such that

$$
|\mathrm{Rm}| \leq \frac{C}{\psi^{2}}
$$


Simon [13, Lemma 2.3] reached the same conlusion in his setting.

Proof. We note first that by Proposition 3.2 the quantity $a=\psi^{2}\left(K_{1}-K_{0}\right)$ remains uniformly bounded.

Since $\psi_{s}$ is bounded, the quantity $\psi^{2} K_{0}=1-\psi_{s}^{2}$ must remain bounded. Hence $\psi^{2} K_{1}=\psi^{2} K_{0}+a$ clearly also remains bounded. The lemma follows via $|\mathrm{Rm}|^{2}=n(n-1) K_{1}^{2}+2 n K_{0}^{2}$.

We now consider a solution $g(t)=(d s)^{2}+\psi(x, t)^{2} \hat{g}$ of Ricci flow and we let $x_{*}(t)$ be the right-most bump (i.e. $x_{*}(t)=\max \left\{x \mid \psi_{x}(x, t)=0\right\}$ ). By Proposition 5.3 we may assume that $\left|\left(\psi^{2}\right)_{t}\right| \leq C_{0}$ for some constant $C_{0}<\infty$. Since

$$
\frac{d}{d t} \psi\left(x_{*}(t), t\right)^{2}=\left(\psi^{2}\right)_{t}\left(x_{*}(t), t\right)+2 \psi \psi_{x} \frac{d x_{*}(t)}{d t}=\left(\psi^{2}\right)_{t}\left(x_{*}(t), t\right)
$$

is also bounded by $C_{0}$ we conclude that

$$
D=\lim _{t \nearrow T} \psi\left(x_{*}(t), t\right)
$$

exists.

Lemma 7.2. If $D>0$, no singularity occurs on the cap $\left(\left(x_{*}(t), 1\right) \times S^{n}(1)\right) \cup$ $\left\{P_{+}\right\}$.

Proof. By Proposition 5.3, we may let $C_{0}$ be an upper bound for $\left|\left(\psi^{2}\right)_{t}\right|$. We choose $t_{1} \in(0, T)$ so that $C_{0}\left(T-t_{1}\right)<D^{2} / 8$. Then we have

$$
\psi\left(x_{*}(t), t\right)^{2} \geq D^{2}-C_{0}(T-t)>\frac{7}{8} D^{2}
$$

for all $t \in\left[t_{1}, T\right)$. Next, let $x_{1}$ be the unique solution of $\psi\left(x_{1}, t_{1}\right)^{2}=\frac{3}{4} D^{2}$ in the interval $\left[x_{*}\left(t_{1}\right), 1\right]$. Our bound on $\left(\psi^{2}\right)_{t}$ implies for all $t \in\left[t_{1}, T\right)$ that

$$
\psi\left(x_{1}, t\right)^{2} \leq \frac{3}{4} D^{2}+C_{0}\left(t-t_{1}\right)<\frac{7}{8} D^{2}<\psi\left(x_{*}(t), t\right)^{2} .
$$

Thus we have $x_{*}(t)<x_{1}<1$ for all $t \in\left[t_{1}, T\right)$, and consequently $\psi_{s}<0$ and $\psi_{s s}<0$ on the interval $\left[x_{1}, 1\right]$ for all $t \in\left[t_{1}, T\right)$. It follows that the distance

$$
d_{1}(t)=s(1, t)-s\left(x_{1}, t\right)
$$

from $\left(x_{1}, t\right)$ to the pole $P_{+}$is decreasing in time. Indeed,

$$
d_{1}^{\prime}(t)=\int_{x_{1}}^{1} \frac{n \psi_{s s}}{\psi} d s<0 .
$$

Next let $x_{2} \in\left(x_{1}, 1\right)$ be defined by $\psi\left(x_{2}, t_{1}\right)^{2}=\frac{3}{8} D^{2}$. Then for $t \in\left[t_{1}, T\right)$ we have

$$
\psi\left(x_{2}, t\right)^{2} \leq \frac{3}{8} D^{2}+C_{0}\left(T-t_{1}\right)<\frac{1}{2} D^{2},
$$

and

$$
\psi\left(x_{1}, t\right)^{2} \geq \frac{3}{4} D^{2}-C_{0}\left(T-t_{1}\right)>\frac{5}{8} D^{2} .
$$


Thus $\psi\left(x_{1}, t\right)^{2}-\psi\left(x_{2}, t\right)^{2} \geq D^{2} / 8$, and hence, crudely estimating $\psi\left(x_{1}, t\right)+$ $\psi\left(x_{2}, t\right)$ from below by

$$
\psi\left(x_{2}, t\right) \geq \sqrt{\frac{3}{8} D^{2}-C_{0}\left(T-t_{1}\right)} \geq \sqrt{D^{2} / 4}=D / 2,
$$

we have

$$
\psi\left(x_{1}, t\right)-\psi\left(x_{2}, t\right) \geq \frac{D^{2} / 8}{D / 2}=D / 4
$$

Concavity (i.e. $\psi_{s s}<0$ ) implies that for $x \in\left[x_{2}, 1\right)$ one has

$$
-\psi_{s}>\frac{\psi\left(x_{1}, t\right)-\psi(x, t)}{s\left(x_{1}, t\right)-s(x, t)}>\frac{D / 4}{s\left(x_{1}, t_{1}\right)-s\left(1, t_{1}\right)} \equiv \delta
$$

At this point we once again consider the quantity $a=\psi \psi_{s s}-\psi_{s}^{2}+1$ from $\S 3.3$. We found that $L(a)=0$, where $L$ is the differential operator

$$
L=\partial_{t}-\partial_{s}^{2}-(n-4) \frac{\psi_{s}}{\psi} \partial_{s}+4(n-1) \frac{\psi_{s}^{2}}{\psi^{2}} .
$$

We also found that the quantity $u=\psi^{\varkappa}$ satisfies

$$
L(u)=(4-\varkappa) \frac{\psi_{s}}{\psi} u_{s}+\frac{n-1}{\psi^{2}}\left(4 \psi_{s}^{2}-\varkappa\right) u=(4-\varkappa) \psi^{\varkappa-2} \psi_{s}^{2}+\frac{n-1}{\psi^{2}}\left(4 \psi_{s}^{2}-\varkappa\right) u .
$$

In the region $Q_{2}=\left[x_{2}, 1\right) \times\left[t_{1}, T\right)$ we have $\left|\psi_{s}\right| \geq \delta$. If we choose $\varkappa<4 \delta^{2}<4$, then we have $L(u)>0$ in $Q_{2}$.

By the maximum principle we then have $|a| \leq C u$ in $Q_{2}$, for some constant $C<\infty$. Indeed, the quantity $|a| / u$ must attain its maximum $(C)$ on the parabolic boundary of $Q_{2}$. At the left end $\left(x=x_{2}\right)$ we have $u=\psi^{\varkappa} \geq(D / 64)^{\varkappa}$, while $|a|$ is bounded by $\sup |a(\cdot, 0)|$ (Corollary 3.2). At the other vertical side of $Q_{2}$, i.e. at $x=1$, we have $a=0$. Since $a$ is smooth, this implies $a=\mathcal{O}(s(1, t)-s(x, t))$. On the other hand $u=\psi^{\varkappa}$ with $\psi_{s}=-1$ at $x=1$ implies that $\lim _{x}{ }_{1}|a / u|=0$ holds for all $t<T$. Finally, at $t=t_{1}$ the quantity $|a| / u$ is continuous for $x_{2} \leq x<1$, while we have just verified that $|a| / u \rightarrow 0$ as $x \nearrow 1$. Thus $|a| / u$ is bounded on the parabolic boundary of $Q_{2}$, and hence bounded on $Q_{2}$.

We now go through a blow-up argument. Let $\mathcal{B}_{2}$ denote the portion of our manifold $S^{n+1}$ where $x \geq x_{2}$. Then we have a solution $g(t)$ to Ricci flow on $\mathcal{B}_{2}$, defined for $t \in\left[t_{1}, T\right)$. This solution has $\mathrm{Rc} \geq 0$.

Because of spherical symmetry $\mathcal{B}_{2}$ is a geodesic ball in $\left(S^{n+1}, g(t)\right)$. Its radius is bounded from above by $d_{1}(t)$. Since $\psi=\psi\left(x_{2}, t\right) \geq D / 2$ on the boundary of $\mathcal{B}_{2}$, it follows from $\left|\psi_{s}\right| \leq 1$ that the radius of $\mathcal{B}_{2}$ is bounded from below by $D / 2$.

Assume that the sectional curvatures of the metrics $g(t)$ on $\mathcal{B}_{2}$ are not bounded as $t \nearrow T$. Then there is a sequence $P_{k} \in \mathcal{B}_{2}, t_{k} \in\left[t_{2}, T\right)$ with $\left|\operatorname{Rm}\left(P_{k}, t_{k}\right)\right| \rightarrow \infty$ as $k \rightarrow \infty$. We may choose this sequence so that

$$
\sup _{Q \in \mathcal{B}_{2}}|\operatorname{Rm}(Q, t)|=\left|\operatorname{Rm}\left(P_{k}, t_{k}\right)\right|
$$


holds for $t \leq t_{k}$. Writing $x_{k}$ for the $x$ coordinate of $P_{k}$ we note that $\psi\left(x_{k}, t_{k}\right) \rightarrow 0$, and from $\left|\psi_{s}\right| \geq \delta$ we conclude

$$
\lim _{k \rightarrow \infty} d_{t_{k}}\left(P_{k}, P_{+}\right)=0
$$

where $d_{t}$ is the distance measured with the metric $g(t)$.

Define $\epsilon_{k}=\left|\operatorname{Rm}\left(P_{k}, t_{k}\right)\right|^{-1 / 2}$, and introduce rescaled metrics

$$
g_{k}(t)=\frac{1}{\epsilon_{k}^{2}} g\left(t_{k}+\epsilon_{k}^{2} t\right)
$$

Let $C_{1}$ be the constant from Lemma 7.1 for which $|\mathrm{Rm}| \leq C_{1} \psi^{-2}$ holds. Then we have

$$
\psi\left(x_{k}, t_{k}\right) \leq \sqrt{C}_{1} \epsilon_{k}
$$

and, using $\left|\psi_{s}\right| \geq \delta$ again,

$$
d_{t_{k}}\left(P_{k}, P_{+}\right) \leq \frac{C_{1}}{\delta} \epsilon_{k}
$$

The distance from $P_{k}$ to the pole $P_{+}$measured in the rescaled metric $g_{k}(0)=$ $\epsilon_{k}^{-2} g\left(t_{k}\right)$ is therefore at most $C_{1} / \delta$. In particular, this distance is uniformly bounded.

Translating to the rescaled metric we find that $g_{k}(t)$ is a solution of Ricci flow defined for $t \in\left(-\epsilon_{k}^{-2} t_{k}, 0\right]$ on the region $\mathcal{B}_{2}$. The Riemann curvature of $g_{k}$ is uniformly bounded by $|\mathrm{Rm}| \leq 1$, with equality attained at $P_{k}$ at $t=0$. One may then extract a convergent subsequence whose limit is an "ancient solution" $g_{\infty}(t)$ of the Ricci flow on $\mathbb{R}^{n+1} \times(-\infty, 0]$ with uniformly bounded sectional curvatures, and nonzero sectional curvature at $t=0$ and at some point $P_{*}$ whose distance to the origin is at most $C_{1} / \delta$.

We introduce the radial coordinate $r=r\left(x, t_{k}\right)=\epsilon_{k}^{-1}\left(s\left(1, t_{k}\right)-s\left(x, t_{k}\right)\right)$. The metric $g_{k}(0)=\epsilon_{k}^{-2} g\left(t_{k}\right)$ seen through the exponential map at $P_{+}$is given by

$$
g_{k}(0)=(d r)^{2}+\Psi_{k}(r)^{2} \hat{g}, \text { with } \Psi_{k}(r)=\epsilon_{k}^{-1} \psi\left(s\left(1, t_{k}\right)-\epsilon_{k} r, t_{k}\right) .
$$

The metrics $g_{k}$ converge in $C^{\infty}$ on regions $r \leq R$ for any finite $R$, and the functions $\Psi_{k}$ hence also converge in $C^{\infty}$ on any interval $[0, R]$. The scale invariant quantity $a$ is given by $a=\psi \psi_{s s}-\psi_{s}^{2}+1=\Psi \Psi_{r r}-\Psi_{r}^{2}+1$, and it satisfies $|a| \leq C \psi^{\varkappa} \leq C \epsilon_{k}^{\varkappa} \Psi_{k}^{\varkappa}$. Thus we find that the limit $\Psi_{\infty}=\lim \Psi_{k}$ satisfies

$$
\Psi \Psi_{r r}-\Psi_{r}^{2}+1=0
$$

Hence for some $\lambda, \mu$ one has

$$
\Psi(r ; \lambda, \mu)= \begin{cases}\frac{1}{\lambda} \sin \lambda(r-\mu), & \lambda<\infty \\ r-\mu & \lambda=\infty\end{cases}
$$

Since $-\Psi_{r}=-\psi_{s} \in[\delta, 1]$ cannot vanish, the only valid solution is the one with $\lambda=\infty, \mu=0$, i.e. $\Psi_{\infty}(r)=r$. But then the limiting metric $g_{\infty}=\lim g_{k}(0)$ is $(d r)^{2}+r^{2} \hat{g}$, i.e. $g_{\infty}$ is the flat Euclidean metric. This is again impossible. 


\section{Construction of the initial metric}

In this section we construct a metric $g_{0}=(d s)^{2}+\Psi(s)^{2} \hat{g}$ which satisfies $\left|\Psi^{\prime}(s)\right| \leq 1, R>0$, and which extends to a smooth metric on $S^{n+1}$. We will show that the solution of Ricci flow starting at $g_{0}$ has a neck pinch, i.e. $r_{\min }(t) \rightarrow 0$, but the diameter of $\left(S^{n+1}, g(t)\right)$ does not shrink to zero.

The particular metric we construct here is obtained from the standard unit sphere $S^{n+1}$ whose metric is $\tilde{g}=(d s)^{2}+(\cos s)^{2} \hat{g}$, by removing a (large) neighborhood of the equator and replacing it with a narrow neck.

\subsection{A neck with positive curvature.}

Lemma 8.1. Consider the function

$$
W(s)=\sqrt{A+B s^{2}},
$$

where $A>0$ and we require $0<B<1$ for $n \geq 3$ and $0<B<1 / 2$ for $n=2$.

Then the scalar curvature of the metric

$$
G=(d s)^{2}+W(s)^{2} \hat{g}
$$

on $\mathbb{R} \times S^{n}$ is positive.

The scale invariant measure of curvature pinching, $a=W(s) W^{\prime \prime}(s)-W^{\prime}(s)^{2}+$ 1 , is bounded by

$$
1-B<a<1+B
$$

Proof. A simple computation shows

$$
\begin{aligned}
\frac{W(s)^{2}}{n} \cdot R & =-2 W(s) W^{\prime \prime}(s)+(n-1)\left\{1-W^{\prime}(s)^{2}\right\} \\
& =n-1-2 B+(3-n) \frac{B^{2} s^{2}}{W}(s)^{2} .
\end{aligned}
$$

Since $B s^{2}<A+B s^{2}=W(s)^{2}$ we find for $n \geq 3$

$$
\frac{W(s)^{2}}{n} \cdot R \geq n-1-2 B+3-n=2(1-B) .
$$

For $n=2$ we get

$$
\frac{W(s)^{2}}{n} \cdot R \geq n-1-2 B=1-2 B
$$

To estimate $a$ we write $a=W W_{s s}-W_{s}^{2}+1=\frac{1}{2}\left(W^{2}\right)_{s s}-2 W_{s}^{2}+1$, which yields

$$
a=B-2 \frac{B^{2} s^{2}}{A+B s^{2}}+1
$$

This implies $1-B<a<1+B$. 
8.2. An initial metric leading to a neck pinch. For any $A>0, B \in(0,1)$ (or $B \in(0,1 / 2)$ when $n=2$ ) we define

$$
\hat{\psi}(s)=\min \{W(s), \cos s\}= \begin{cases}W(s) & |s| \leq s_{A, B} \\ \cos s & s_{A, B}<|s| \leq \pi / 2,\end{cases}
$$

where $s_{A, B}$ is the unique positive solution of $\cos s=\sqrt{A+B s^{2}}$. This piecewise smooth function clearly satisfies $\left|\hat{\psi}^{\prime}(s)\right| \leq 1$ and $R>0$ for all $s \neq \pm s_{A, B}$.

We now smooth out the corner which $\hat{\psi}$ has at $s_{A, B}$. First we construct a new function $\breve{\psi}$ which coincides with $\hat{\psi}$ outside a small interval $I_{\epsilon}=\left(s_{A, B}-\epsilon, s_{A, B}+\epsilon\right)$

and has $\breve{\psi}_{s s}$ constant in $I_{\epsilon}$. The value of the constant is determined by requiring $\breve{\psi}$ to be a $C^{1}$ function. Since $\hat{\psi}$ switches from increasing to decreasing at $s_{A, B}$, we will have $\breve{\psi}_{s s}<0$ in $I_{\epsilon}$. Moreover, we will also have $\left|\breve{\psi}_{s}\right|<1$ in $I_{\epsilon}$, and hence the metric $(d s)^{2}+\breve{\psi}^{2} \hat{g}$ will have $R>0$ everywhere.

The function $\breve{\psi}$ is $C^{1}$, and its second derivative $\breve{\psi}_{s s}$ has simple jump discontinuities at $s_{A, B} \pm \epsilon$ so we may smooth it in an arbitrarily small neighborhood of $s_{A, B} \pm \epsilon$ in such a way that the smoothed $\left(C^{\infty}\right)$ function, which we will call $\psi_{A, B}(s)$, satisfies $R>0$ everywhere and coincides with $\hat{\psi}$ outside of $I_{2 \epsilon}$.

It is not hard to see that there is some small $\alpha>0$ for which one can execute the smoothing of the $\hat{\psi}=\hat{\psi}_{A, B}$ for all $A \in(0, \alpha)$ in such a way that the $\psi_{A, B}$ coincide with $\hat{\psi}$ in the interval $|s| \leq \alpha$, and such that the derivatives $\psi_{A, B}^{\prime \prime}(s)$ are bounded for $|s| \geq \alpha$, uniformly in $A \in(0, \alpha)$. If this is done, then we obtain a family of initial metrics $g_{A, B}=(d s)^{2}+\psi_{A, B}(s)^{2} \hat{g}$

- with positive scalar curvature,

- which satisfy $\left|\psi_{s}\right| \leq 1$,

- which have a neck of radius $r_{\min }(0)=\sqrt{A}$,

- which have a bump of height at least $\hat{\psi}_{A, B}(\alpha)>\sqrt{B} \alpha$,

- for which $|a| \leq C$ for some constant $C<\infty$ which does not depend on $A \in(0, \alpha)$.

Lemma 6.1 implies that the solution to Ricci flow starting from $g_{A, B}$ must lose its neck before $t_{*}=r_{\min }(0)^{2} /(n-1)=A /(n-1)$. On the other hand, the solution will have a bump at some $x_{*}(t)$; and since $|a|$ is uniformly bounded on all solutions under consideration, the height of this bump will be bounded from below by

$$
\psi\left(x_{*}(t), t\right)^{2} \geq \alpha \sqrt{B}-C t \geq \alpha \sqrt{B}-C A /(n-1) .
$$

If $A$ is small enough, then the neck must disappear before the bump can vanish, and thus $r_{\min }(t) \rightarrow 0$.

\section{Cylindrical Asymptotics}

In this section we consider a maximal solution $g(\cdot, t)=(d s)^{2}+\psi^{2} \hat{g}$ to the Ricci flow, defined for $0 \leq t<T$. 
We will assume that $\left|\psi_{s}\right| \leq 1$ and $R \geq 0$ hold initially and hence for all time. We will also assume that the solution always has at least one neck. Let $x_{-}(t)$ and $x_{+}(t)$ be the left- and right-most bumps, respectively, and write $\mathcal{W}(t)=$ $\left[x_{-}(t), x_{+}(t)\right]$.

For each $t \in[0, T)$ and $x \in \mathcal{W}(t)$ one has $\psi(x, t) \geq r_{\min }(t)$ so that the sectional curvature $L=K_{1}=\left(1-\psi_{s}^{2}\right) / \psi^{2}$ is bounded from above on $\mathcal{W}(t)$ by $r_{\min }(t)^{-2}$.

By the strong maximum principle we may assume that $\sup _{\mathcal{W}(t)}\left|\psi_{s}(\cdot, t)\right|<1$ so that $L$ is also bounded from below. Define

$$
L_{\min }(t)=\inf _{x \in \mathcal{W}(t)} L(x, t) .
$$

The evolution equation (23a) implies that $L_{\min }(t)$ is nondecreasing.

Recall that in equation (25) in Section 3.4, we introduced $F=\frac{K}{L} \log L$, which evolves according to (26).

Lemma 9.1. For all $t \in[0, T), x \in \mathcal{W}(t)$ the scaling-invariant quantity

$$
\hat{F}(x, t)=\frac{K(x, t)}{L(x, t)}\left(\log L(x, t)+2-\log L_{\min }(0)\right)
$$

satisfies

$$
\sup _{\mathcal{W}(t)} \hat{F}(\cdot, t) \leq \max \left\{n-1, \sup _{\mathcal{W}(0)} \hat{F}(\cdot, 0)\right\} .
$$

We note that $\hat{F}$ is invariant under simultaneous rescaling of the metric $g \mapsto \lambda g$ and time $t \mapsto \lambda t$.

Proof. We first deal with the case in which $L_{\min }(0) \geq e^{2}$. Since $L_{\min }(t)$ is nondecreasing we also have $L_{\min }(t) \geq 2$ in this case.

Our proof will proceed by applying the maximum principle to equation (26) which $F$ satisfies. We will apply the maximum principle in the region where $F \geq n-1$. Since we are currently assuming $L \geq e^{2}>1, F$ and $K$ have the same sign, so that $F \geq n-1$ implies $K \geq 0$.

We use our assumption of positivity of the scalar curvature to conclude $0 \leq$ $R=n[-2 K+(n-1) L]$ and hence

$$
K \leq \frac{n-1}{2} L .
$$

Using $L>e^{2}>e$, we conclude that the coefficient $P$ in (26) satisfies

$$
P \geq(n-1) \log L-(n-1)(\log L-1)=n-1 .
$$

Thus when $K>0$ and $L>e^{2}$, (26) implies the differential inequality

$$
F_{t} \leq \Delta F+2\left(\frac{\log L-1}{L \log L}\right) L_{s} F_{s}+2 Q K .
$$

But if $F \geq n-1$, then we have

$$
Q \leq-\frac{K^{2}}{L^{2}}(\log L-1) \leq 0
$$


and hence

$$
F_{t} \leq \Delta F+2\left(\frac{\log L-1}{L \log L}\right) L_{s} F_{s}
$$

We conclude that if $L_{\min }(0) \geq e^{2}$ then $\sup F(\cdot, t)$ cannot increase whenever it exceeds $n-1$.

To complete the proof we must deal with possibility that $L_{\min }(0)<e^{2}$. Should this occur, then we rescale both time and the metric, i.e. we consider $\tilde{g}(t)=$ $\lambda g(t / \lambda)$ which again satisfies the Ricci flow equation. Denoting its sectional curvatures by $\tilde{K}$ and $\tilde{L}$ we have $\tilde{L}_{\min }=\lambda^{-1} L_{\min }, \tilde{K}=\lambda^{-1} K$. Thus if we choose $\lambda=e^{-2} L_{\min }(0)$, then $\tilde{L}(0) \geq e^{2}$, and the preceding arguments apply to the metric $\tilde{g}$. Hence $\max \{n-1, \tilde{F}\}$ is nonincreasing. In terms of the original metric we have found that

$$
\max \left\{n-1, \frac{K}{L} \log \left(\frac{e^{2}}{L_{\min }(0)} L\right)\right\}
$$

does not increase with time, as claimed.

9.1. Convergence to a cylinder. For $t \in[0, T)$ close to $T$, we choose $x_{0}(t) \in$ $\mathcal{W}(t)$ so that $\psi\left(x_{0}(t), t\right)=r_{\min }(t)$. Let

$$
\sigma=s(x, t)-s\left(x_{0}(t), t\right) \text {. }
$$

We will now prove

Lemma 9.2. There are constants $\delta>0$ and $C<\infty$ such that for $t$ sufficiently close to $T$ one has

$$
1 \leq \frac{\psi(x, t)}{r} \leq 1+\frac{C}{-\log r}\left(\frac{\sigma}{r}\right)^{2}
$$

for $|\sigma| \leq 2 r \sqrt{-\log r}$, and

$$
\frac{\psi(x, t)}{r} \leq C \frac{\sigma}{r \sqrt{-\log r}} \log \frac{\sigma}{r \sqrt{-\log r}}
$$

for $2 r \sqrt{-\log r} \leq \sigma \leq r^{1-\delta}$. Here we have written $r=r_{\min }(t)$, for short.

Proof. Choose a small number $\beta>0$.

We regard $t$ as fixed, and consider the neighborhood of the neck $x_{0}(t)$ in which $\psi \leq \beta$ and $\left|\psi_{s}\right|<\beta$. In this region one always has $L \geq\left(1-\beta^{2}\right) / \beta^{2}$, so that $\left|2-\log L_{\min }(0)\right| \leq C L$ for some constant $C<\infty$. By Lemma 9.1, we may assume that $\frac{K}{L} \log L \leq C$ for some $C<\infty$. In terms of $\psi$, this means

$$
\frac{\psi_{s s}}{\psi} \leq \frac{1-\psi_{s}^{2}}{\psi^{2}} \cdot \frac{C}{\log \frac{1-\psi_{s}^{2}}{\psi^{2}}},
$$

and thus

$$
\psi \psi_{s s} \leq C \frac{1-\psi_{s}^{2}}{\log \left(1-\psi_{s}^{2}\right)-2 \log \psi}
$$


whence

$$
\frac{\psi \psi_{s s}}{1-\psi_{s}^{2}} \leq \frac{C}{-2 \log \psi} \cdot \frac{1}{1-\frac{1}{2} \frac{\log \left(1-\psi_{s}^{2}\right)}{\log \psi}} .
$$

Since we only considering the region where $\psi \leq \beta$ and $\left|\psi_{s}\right| \leq \beta$, we have

$$
1-\frac{1}{2} \frac{\log \left(1-\psi_{s}^{2}\right)}{\log \psi} \geq 1-\frac{1}{2} \frac{\log \left(1-\beta^{2}\right)}{\log \beta} .
$$

By choosing $\beta$ small enough we can make the righthand side $\geq \frac{1}{2}$, so that we get

$$
\frac{\psi \psi_{s s}}{1-\psi_{s}^{2}} \leq \frac{C}{-\log \psi}
$$

We now further restrict our attention to the region to the right of the neck, where $\psi_{s}>0$, and where we may choose the radius $\psi$ as a coordinate; i.e. we regard all quantities as functions of $\psi$. Then we have

$$
-\frac{d \log \left(1-\psi_{s}^{2}\right)}{d \log \psi}=\frac{2 \psi_{s} \psi_{s s}}{1-\psi_{s}^{2}} \cdot \frac{\psi}{\psi_{s}} \leq \frac{C}{-\log \psi} .
$$

Integrate this from the neck, where $\psi=r_{\min }(t)=r$ and $\psi_{s}=0$, to an arbitrary point. One gets

$$
-\log \left(1-\psi_{s}^{2}\right) \leq \int_{u=r}^{\psi} \frac{C}{-\log u} d \log u=C \log \frac{\log r}{\log \psi} .
$$

Using the calculus inequalities $x \leq-\log (1-x)$ and $\log x \leq x-1$, we arrive at

$$
\psi_{s}^{2} \leq C \log \frac{\log r}{\log \psi} \leq C\left(\frac{\log r}{\log \psi}-1\right) .
$$

We are always assuming that $\left|\psi_{s}\right| \leq \beta$, so this last inequality will only be useful if the righthand side is no more than $\beta^{2}$. Henceforth we assume that

$$
r \leq \psi \leq\left(\frac{1}{r}\right)^{\beta^{2} / 2 C} r
$$

Using $e^{-\beta^{2} / C} \leq 1-\beta^{2} / 2 C$ for small $\beta$, one finds that (35) and (34) imply $\left|\psi_{s}\right| \leq \beta$ and $\psi<\beta$, as required.

Integrating once again, we get

$$
\sqrt{C} \sigma \geq \int_{r}^{\psi} \frac{d u}{\sqrt{\frac{\log r}{\log u}-1}}
$$

Substitute $u=r v$. It follows from $r \leq u \leq \psi$ that $1 \leq v \leq \psi / r$, and by (35), that $0 \leq \log v \leq-\frac{\beta^{2}}{2 C} \log r$. Hence we get

$$
\sqrt{C} \frac{\sigma}{r} \geq \int_{1}^{\psi / r} \sqrt{-\log r-\log v} \frac{d v}{\sqrt{\log v}} \geq \frac{1}{2} \sqrt{-\log r} \int_{1}^{\psi / r} \frac{d v}{\sqrt{\log v}} .
$$


Proposition 9.3. The function $Z:[0, \infty) \rightarrow[1, \infty)$ defined by

$$
x=\int_{1}^{Z(x)} \frac{d u}{\sqrt{\log u}}
$$

is monotone increasing and satisfies

$$
Z(x)=\left\{\begin{array}{ll}
1+\frac{1}{2} x^{2}+o\left(x^{2}\right) & (x \searrow 0) \\
(1+o(1)) x \sqrt{\log x} & (x \rightarrow \infty)
\end{array} .\right.
$$

In particular, we have, for some constant $C<\infty$,

$$
Z(x) \leq \begin{cases}1+C x^{2} & (0 \leq x \leq 2) \\ C x \sqrt{\log x} & (x \geq 2)\end{cases}
$$

We leave the proof to the reader. Our last estimate (36) can be recast as $\psi / r \leq Z(C \sigma / r \sqrt{-\log r})$, which combined with the estimate of $Z(x)$ for $x \leq 2$ gives (32).

For larger $\sigma$ we get

$$
\frac{\psi}{r} \leq C \frac{\sigma}{r \sqrt{-\log r}} \sqrt{\log \frac{\sigma}{r \sqrt{-\log r}}}
$$

which is exactly (33). This estimate will be valid when $\sigma \geq 2 r \sqrt{-\log r}$, while (35) must also be satisfied. Using $C \sqrt{-\log r}=r^{o(1)}$ we find that (33) will hold if $\psi / r \leq(1 / r)^{\beta^{2} / 2 C+o(1)}$.

9.2. The type-I blow-up. Let $g(t)$ be the solution of Ricci flow considered above. We know by Lemma 7.2 that the curvature stays bounded on the polar caps, while Lemma 7.1 applied to the waist $\mathcal{W}(t)$ gives us the upper bound $|\mathrm{Rm}| \leq C r_{\min }(t)^{-2}$. Since $r_{\min }(t) \geq C \sqrt{T-t}$, we find that $|\mathrm{Rm}| \leq C(T-t)^{-1}$; i.e. the singularity is of "type-I," namely "fast-forming."

One can therefore construct a type-I blow-up by considering the metrics $\tilde{g}(t)=$ $(T-t)^{-1} g(t)$. Near a neck, these will converge to an ancient solution of Ricci flow; and in view of Lemma 9.2, this ancient solution must be the cylinder solution. It follows that

$$
r_{\min }(t)=(1+o(1)) \sqrt{2(n-1)(T-t)} .
$$

In view of this, Lemma 9.2 implies

Lemma 9.4. There are constants $\delta>0$ and $C<\infty$ such that for $t$ sufficiently close to $T$ one has

$$
1+o(1) \leq \frac{\psi(x, t)}{\sqrt{2(n-1)(T-t)}} \leq 1+\frac{C}{-\log (T-t)} \frac{\sigma^{2}}{T-t}
$$

for $|\sigma| \leq 2 \sqrt{-(T-t) \log (T-t)}$, and

$$
\frac{\psi(x, t)}{\sqrt{T-t}} \leq C \frac{\sigma}{\sqrt{-(T-t) \log (T-t)}} \log \frac{\sigma}{\sqrt{-(T-t) \log (T-t)}}
$$


for $2 \sqrt{-(T-t) \log (T-t)} \leq \sigma \leq(T-t)^{(1-\delta) / 2}$.

\section{Equatorial pinching}

In this section, we consider the special case of a reflection-invariant metric on $S^{n+1}$ with a single symmetric neck at $x=0$ and two bumps. In this case, we prove that the neckpinch singularity will occur only on the totally-geodesic hypersurface $\{0\} \times S^{n}$, unless the diameter of the sphere $S^{n+1}$ becomes infinite as the singularity time is approached. (We do not expect the latter alternative to occur.) Our method will be to construct a family of subsolutions $\underline{v}$ for $v=\psi_{s}$.

As in Section 5.4, let $x_{*}(t)$ denote the location of the right-hand bump (the unique point in $(0,1)$ where $\psi_{\max }(t)$ is attained). As in Section 7 , let $D=$ $\lim _{t \nearrow T} \psi\left(x_{*}(t), t\right)$ denote the final height of that bump. By the construction in Section 8 , we may assume that $D>0$. Define the function

$$
\rho(t)=n \int_{0}^{t} \int_{0}^{D}\left(\frac{\psi_{s}}{\psi}\right)^{2} d s d t
$$

noting that $\rho$ is monotone increasing in time, so that $\rho(T)=\lim _{t \nearrow T} \rho(t)$ exists.

Now let $s_{*}(t)=s\left(x_{*}(t), t\right)$ denote the distance from the equator to the righthand bump. By Proposition 5.1, one has $\left|\psi_{s}\right| \leq 1$; and because $\partial_{t} \psi_{\max } \leq$ $-(n-1) / \psi_{\max }$, one has $\psi\left(s_{*}(t), t\right)>D$ for all $t \in[0, T)$. Together, these results imply that $s_{*}(t)>D$ for all $t \in[0, T)$. Let $x_{D}(t)$ denote the unique point in $\left(0, x_{*}(t)\right)$ such that $s\left(x_{D}(t), t\right)=D$. Notice that for any $s$, the hypothesis of reflection symmetry about $x=0$ lets one integrate by parts to obtain the identity

$$
\frac{\partial s}{\partial t}=n \int_{0}^{x} \frac{\psi_{s s}}{\psi} \frac{\partial s}{\partial x} d x=n\left\{\frac{\psi_{s}}{\psi}+\int_{0}^{s(x)}\left(\frac{\psi_{s}}{\psi}\right)^{2} d s\right\} .
$$

Since Lemma 5.5 implies that $\psi_{s} \geq 0$ when $0 \leq s \leq s_{*}(t)$, one may then estimate at any $\hat{x} \in\left[x_{D}(t), x_{*}(t)\right]$ that

$$
s_{*}(t) \geq s(\hat{x}, t) \geq n \int_{0}^{t} \int_{0}^{s(\hat{x})}\left(\frac{\psi_{s}}{\psi}\right)^{2} d s d t \geq \rho(t) .
$$

In particular, $\rho(t)$ is bounded above by the distance from the equator to the bump.

We are now ready to prove the following "single-point pinching" result:

Lemma 10.1. If the diameter of the solution $g(t)$ remains bounded as $t \nearrow T$, then $\psi(s, T)>0$ for all $0<s<D / 2$.

To establish the lemma, let $t_{0} \in(0, T)$ and $\delta>0$ be given. For $\varepsilon>0$ to be chosen below, define

$$
\underline{v}(s, t)=\varepsilon\left\{s-\left[\rho(t)-\rho\left(t_{0}-\delta\right)\right]\right\} .
$$

By (40), the finite-diameter assumption implies that $\rho(T)<\infty$, hence that

$$
\sup _{t_{0}-\delta<t<T}\left[\rho(t)-\rho\left(t_{0}-\delta\right)\right]
$$


becomes arbitrarily small when $t_{0}-\delta$ is sufficiently close to $T$. The lemma is thus an immediate consequence of

Proposition 10.2. If $\rho(T)<\infty$, then for any $t_{0} \in(0, T)$ and $\delta \in\left(0, t_{0}\right)$, there exists $\varepsilon>0$ such that $v=\psi_{\text {s }}$ satisfies

$$
v(s, t) \geq \underline{v}(s, t)
$$

for all points $0<s<D / 2$ and times $t_{0} \leq t<T$.

Proof. Since $\underline{v}\left(0, t_{0}\right)<0$ and $v\left(s, t_{0}\right)>0$ for all $s \in\left(0, s_{*}\left(t_{0}\right)\right)$, one may choose $\varepsilon_{1}$ such that if $0<\varepsilon<\varepsilon_{1}$, then $v\left(s, t_{0}\right)>\underline{v}\left(s, t_{0}\right)$ whenever $0 \leq s \leq D / 2$. So if the result is false, there will be a first time $\bar{t} \in\left(t_{0}, T\right)$ and a point $\bar{s} \in(0, D / 2)$ such that $v(\bar{s}, \bar{t})=\underline{v}(\bar{s}, \bar{t})$. At $(\bar{s}, \bar{t})$, one then has

$$
v_{t} \leq \underline{v}_{t}=\varepsilon\left(\frac{\partial s}{\partial t}-\rho^{\prime}\right)
$$

as well as $v=\underline{v}, v_{s}=\underline{v}_{s}=\varepsilon$, and $v_{s s} \geq \underline{v}_{s s}=0$. Hence

$$
v_{t}=v_{s s}+\frac{(n-2)}{\psi} v v_{s}+\frac{(n-1)}{\psi^{2}}\left(1-v^{2}\right) v \geq \varepsilon \frac{(n-2)}{\psi} v+\frac{(n-1)\left(1-\underline{v}^{2}\right)}{\psi^{2}} v .
$$

Whenever $0<\varepsilon<\varepsilon_{2}=\sqrt{2} / D$, one has $\underline{v} \leq \varepsilon s<1 / \sqrt{2}$ for all $0 \leq s \leq D / 2$ and $t \in\left[t_{0}, T\right)$. Then because $v \geq 0$ for $s \in(0, D / 2) \subset\left[0, s_{*}(t)\right]$, one estimates at $(\bar{s}, \bar{t})$ that

$$
\begin{aligned}
\underline{v}_{t}-v_{t} & \leq \varepsilon\left(\frac{\partial s}{\partial t}-\rho^{\prime}-\frac{n-2}{\psi} v\right)-(n-1)\left(1-\underline{v}^{2}\right) \frac{v}{\psi^{2}} \\
& \leq \varepsilon\left(\frac{\partial s}{\partial t}-\rho^{\prime}\right)-\frac{n-1}{2} \frac{v}{\psi^{2}} \\
& =\varepsilon n\left[\frac{v}{\psi}-\int_{\bar{s}}^{D}\left(\frac{v}{\psi}\right)^{2} d s\right]-\frac{n-1}{2} \frac{v}{\psi^{2}} \\
& <\frac{v}{\psi}\left[\varepsilon n-\frac{n-1}{2 \psi}\right] .
\end{aligned}
$$

Choose $\varepsilon_{3}<(n-1) /\left[2 n \psi_{\max }(0)\right]$. Then if $0<\varepsilon<\min \left\{\varepsilon_{1}, \varepsilon_{2}, \varepsilon_{3}\right\}$, the consequence $\psi_{t}<0$ of Proposition 5.2 implies the inequality $\underline{v}_{t}-v_{t}<0$. This contradicts (41), hence establishes the result.

\section{References}

[1] S.B. Angenent, The zero set of a solution of a parabolic equation, Journal für die reine and angewandte Mathematik 390 (1988), 79-96.

[2] S. Altschuler, S.B. Angenent, Y. Giga, Mean curvature flow through singularities for surfaces of rotation, J. Geom. Anal. 5 (1995), 293-358.

[3] S.B. Angenent, J.J.L. Velázquez, Degenerate neckpinches in mean curvature flow, J. Reine Angew. Math. 482 (1997), 15-66.

[4] M. Feldman, T. Ilmanen, D. Knopf, Rotationally symmetric shrinking and expanding gradient Kähler-Ricci solitons, J. Geom. Anal. 65 (2003), 169-209. 
[5] S. Filippas, R.V. Kohn, Refined asymptotics for the blowup of $u_{t}-\Delta u=u^{p}$. Comm. Pure Appl. Math. 45 (1992), 821-869.

[6] Y. Giga, R.V. Kohn, Asymptotically self-similar blow-up of semilinear heat equations. Comm. Pure Appl. Math. 38 (1985), 297-319.

[7] Y. Giga, R.V. Kohn, Characterizing blowup using similarity variables. Indiana Univ. Math. J. 36 (1987), 1-40.

[8] Y. Giga, R.V. Kohn, Nondegeneracy of blowup for semilinear heat equations. Comm. Pure Appl. Math. 42 (1989), 845-884.

[9] R.S. Hamilton, Three-manifolds with positive Ricci curvature. J. Differential Geom. 17 (1982), 255-306.

[10] R.S. Hamilton, The formation of singularities in the Ricci flow. Surveys in differential geometry, Vol. II (Cambridge, MA, 1993), 7-136, Internat. Press, Cambridge, MA, 1995.

[11] R.S. Hamilton, Four-manifolds with positive isotropic curvature, Comm. Anal. Geom. 5 (1997), 1-92.

[12] G. Huisken, Asymptotic behavior for singularities of the mean curvature flow. J. Differential Geom. 31 (1990), 285-299.

[13] M. Simon, A class of Riemannian manifolds that pinch when evolved by Ricci flow, Manuscripta Math. 101 (2000), 89-114.

[14] G. Perelman, The entropy formula for the Ricci flow and its geometric applications. arXiv:math.DG/0211159.

[15] G. Perelman, Ricci flow with surgery on three-manifolds. arXiv:math.DG/0303109.

(Sigurd Angenent) University of Wisconsin - Madison

E-mail address: angenent@math.wisc.edu

$U R L:$ http: //www.math.wisc.edu/ ${ }^{\sim}$ angenent

(Dan Knopf) The University of IowA

E-mail address: dknopf@math.uiowa.edu

$U R L:$ http://www.math.uiowa.edu/ dknopf 